\section{Ultrasound in phage display: a new approach to nonspecific elution}

\author{
Mojca Lunder¹, Tomaž Bratkovič ${ }^{1}$, Uroš Urleb², Samo Kreft ${ }^{1}$, and Borut \\ Štrukelj ${ }^{1}$ \\ ${ }^{1}$ Faculty of Pharmacy, University of Ljubljana and 2Lek Pharmaceuticals, \\ Ljubljana, Slovenia
}

BioTechniques 44:893-900 (June 2008)

doi $10.2144 / 000112759$

Libraries of phage-displayed random peptides are routinely used to identify target-binding peptides. Phages are commonly eluted in a nonspecific manner, especially if there are no available ligands of the particular target to use as competitors. However, the present study clearly demonstrates that nonspecific elution is not always able to break peptide-target interactions. To circumvent this we have developed an improved nonspecific elution strategy that uses ultrasound to release target-bound phages and enables selection of high-affinity clones in a single step.

\section{INTRODUCTION}

Phage display is used to select and optimize peptides or protein domains binding to virtually any protein and sometimes even nonprotein targets (1-3). A typical round of selection involves: (i) target immobilization; (ii) reaction of the library with the target; (iii) removal of unbound phages through multiple washes; and (iv) elution of bound phages from the target followed by their amplification in bacterial cells. Several rounds of screening are performed until the increase in phage output, or binding assays performed with phage pools, indicates that the population of binding phages has been adequately enriched $(4,5)$.

Typically, target-bound virions are released by nonspecific elution with acidic buffer such as $0.2 \mathrm{M}$ glycine- $\mathrm{HCl}$, $\mathrm{pH} 2.2$ (1). Extreme $\mathrm{pH}$ and other means of nonspecific elution (e.g., high ionic strength, reductants such as DTT, specific proteases) tend to loosen the phage-target interaction, or denature or degrade either the target or the phage-displayed peptide. In cases where ligands of particular target are known and available, competitive elution may be preferred. Alternative methods of competitive, but nonspecific, elution involve using the free target molecule as an eluant $(1,6)$ or adding bacterial host directly to the target-bound phages (7).

For a nonspecific elution strategy to result in successful affinity selection, the essential condition is thought to be appropriate washing stringency. Washing protocols can be optimized empirically for every single target. Under controlled washing conditions any target-unrelated binders should be removed, whereas selective binding of phages to the target should not be compromised $(3,7)$.

Most commonly, the concentration of surfactants in the washing buffer is raised and more washing steps are performed in successive rounds of selection when higher stringency works in favor of affinity selection. Ionic strength or $\mathrm{pH}$ can also be varied and even known ligands of the target can be added to the washing buffer (7-10).

Here we show that the main reason for unsuccessful selection of ligands from phage-displayed libraries with nonspecific elution may not be the poorly chosen washing conditions but rather the inability of the nonspecific elution method to break the strongest virion-target interactions.

Sonication has been used to break the antigen-antibody interaction. Digoxin was removed from immobilized anti-digoxin antibody (11). The oscillation of cavitation bubbles produced by high-power ultrasound is known to cause hydrodynamic sheering stress, which aids in dissociation of complexes. To overcome the limitation of nonspecific elution methods in phage display we propose a completely new approach, based on the use of ultrasound.

\section{MATERIALS AND METHODS}

\section{Selection Protocols}

Microtiter plates (StreptaWell, Roche, Mannheim, Germany) precoated and preblocked with streptavidin (Sigma-
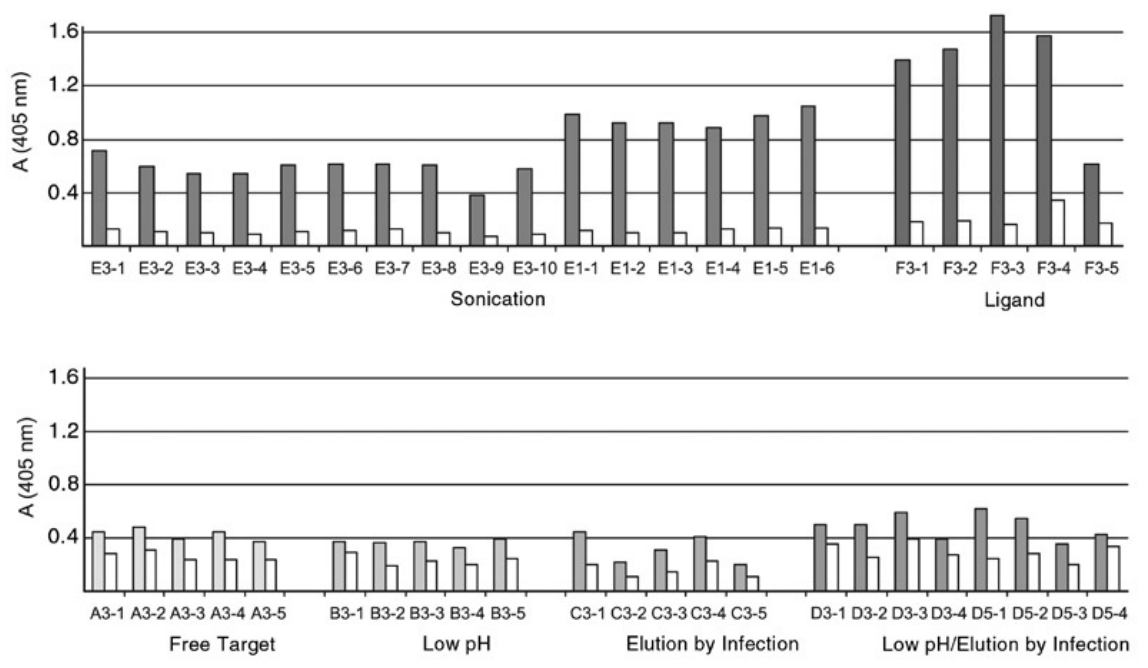

Figure 1. ELISA screening of individual phage clones. Phage clones were amplified by infecting 2 $\mathrm{mL}$ of bacterial culture Escherichia coli ER2738 $\left(\mathrm{OD}_{600} \sim 0.1\right)$ and, after $4.5 \mathrm{~h}$ separated from bacteria by centrifugation, omitting subsequent isolation and determination of phage titer. Prepared suspensions of each phage clone were used in ELISA assay as described in Materials and Methods. A name was assigned to each phage clone, and is composed of the character describing the selection protocol (letters A-E, Table 1), a number of selection rounds performed, and a consecutive number of the clone. The name is consistent with the label of bars representing the absorbance at $405 \mathrm{~nm}$. If the ELISA signal of an individual clone exceeded the blank by at least fourfold, the primary sequence of peptide displayed on that clone was determined. Elution by sonication (Protocol E) and elution with biotin (Protocol F) resulted in enrichment of phages with the highest affinity toward streptavidin. All sequenced phages displayed the same cyclic heptapeptide CGTFAHPQC. Slightly different phage concentrations and interday variability of the ELISA assay are the cause of different OD signals for clones that share the same peptide sequence. In other selection protocols (A-D), the average ELISA signal exceeded the blank by twofold or less, therefore binding was considered to be target-unrelated. 
Aldrich, Steinheim, Germany) were incubated with an aliquot of $2 \times 10^{11}$ phages (random cyclic heptapeptide phage display library, Ph.D.-C7C; New England Biolabs, Beverly, MA, USA), diluted to $100 \mu$ l with PBST $(135 \mathrm{mM} \mathrm{NaCl}, 3 \mathrm{mM}$ $\mathrm{KCl}, 10 \mathrm{mM} \mathrm{Na} 2 \mathrm{HPO}_{4}, 2 \mathrm{mM} \mathrm{KH} \mathrm{PO}_{4}$; $\mathrm{pH}$ 7.4), containing $0.1 \%$ Tween 20 , for $1 \mathrm{~h}$ at room temperature with gentle agitation. Nonbinding phages were discarded by washing the wells with different washing strategies (Table 1). Bound clones were eluted with different elution strategies (Table 1). Washing and elution steps are described in detail below.

Eluted phages were amplified by infecting Escherichia coli ER2738 host cells (New England Biolabs). After $5 \mathrm{~h}$ growth at $37^{\circ} \mathrm{C}$, bacteria were removed by centrifugation $(12,186 \times g)$, and phages in the supernatant were precipitated by addition of $1 / 6$ volume of $\mathrm{PEG} / \mathrm{NaCl}$ solution $(20 \%$ polyethylene glycol-8000, $2.5 \mathrm{M} \mathrm{NaCl}$ ) and overnight incubation at $4^{\circ} \mathrm{C}$. The precipitate was resuspended in a small volume of PBS and amplified eluates were titered to determine phage concentrations. Finally, eluates from the last round of selection were used to infect plated bacterial host cells and 10 plaques were selected at random. Individual phage clones were then grown and purified for analysis.

\section{Washing and Elution Steps in Protocols A-F}

A brief description of washing and elution steps is given in Table 1. In Protocol A, all three selection rounds were performed in the same manner. Following $1 \mathrm{~h}$ hybridization of the phage library with streptavidin, unbound phage clones were removed by washing the microtiter plate surface 10 times with $0.2 \%$ PBST. Next, residual phage clones were eluted at room temperature for $1 \mathrm{~h}$ with $100 \mu \mathrm{l}$ of $100 \mu \mathrm{g} / \mathrm{mL}$ free streptavidin solution to compete the bound phages away from the immobilized target.

In Protocol B, washing and elution conditions intensified in the second and third selection round. In the first round, 30 PBST washings were applied, followed by 35 PBST washings in the second and third selection rounds, to remove the unbound phages. For each PBST washing, 200 $\mu \mathrm{l}$ of $0.2 \%$ PBST buffer were added to the well and shaken for $5 \mathrm{~min}$ at $50 \mathrm{rpm}$ and then discarded. Next, bound phages were eluted with $100 \mu \mathrm{l}$ of $0.2 \mathrm{M}$ glycine$\mathrm{HCl}$ buffer, $\mathrm{pH}$ 2.2. In the first round, 8 consecutive eluates were combined and amplified, and in the second and third rounds 10 were combined and amplified. For each eluate, glycine- $\mathrm{HCl}$ buffer was transferred into the microtiter well and shaken for $5 \mathrm{~min}$ at $100 \mathrm{rpm}$, removed from the microtiter well, and neutralized with $100 \mu \mathrm{l}$ of $200 \mathrm{mM}$ phosphate buffer, $\mathrm{pH}$ 7.5. The same amount of this buffer was used to stabilize the microtiter well surface between glycine-HCl elutions and then added to the eluates.

In Protocol C, washing conditions intensified from the first to the third selection round. The first round was performed with 10 PBST washings, followed by 4 glycine- $\mathrm{HCl}(0.2 \mathrm{M}, \mathrm{pH}$ 2.2) washings. The second round was performed with 20 PBST and 8 glycine-
$\mathrm{HCl}$ washings, and the third round with 35 PBST and 16 glycine- $\mathrm{HCl}$ washings. After the washing step in each round, the remaining phages were eluted by direct bacterial infection. One hundred microliters of $E$. coli ER2738 host cells $\left(\mathrm{OD}_{600}\right.$ $\sim 0.1$ ) were added to the well and shaken at $50 \mathrm{rpm}$ for $1 \mathrm{~h}$. The infected host cells were then transferred to fresh LB medium and further amplified.

Protocol D included five rounds of selection. In each round, the number of washings with PBST was gradually increased from 10 to 35 . Phages were eluted as described in Protocol B. Additionally, remaining phages were eluted by direct bacterial infection. All eluates were combined and amplified.

In Protocol E, elution by sonication was introduced. After 25 washings with PBST and 4 with glycine- $\mathrm{HCl}(0.2 \mathrm{M}$, $\mathrm{pH} 2.2$ ), another $100 \mu \mathrm{l}$ of glycine- $\mathrm{HCl}$ were added to the microtiter plate well (Maxisorp, Nalge Nunc International, Roskilde, Denmark), and the covered plate was immersed in a sonicator water bath $(50 \mathrm{kHz})$ for $10 \mathrm{~min}$. The eluate was transferred into $100 \mu \mathrm{l}$ of neutralizing buffer (200 mM phosphate buffer, $\mathrm{pH}$ 7.5). Three rounds of selection were performed.

Protocol F included three selection rounds. Washing steps were identical to those in Protocol E, followed by elution with $100 \mu \mathrm{l}$ of $0.1 \mathrm{mM}$ biotin for $1 \mathrm{~h}$ at 50 rpm.

Additional biotin elutions were performed to clarify the presence or absence of high-affinity clones in enriched libraries obtained in Protocols A, B and C. After the third round of selection, two further rounds of selection were carried

Table 1. Overview of Selection Protocols

\begin{tabular}{|c|c|c|c|c|c|c|c|}
\hline \multicolumn{2}{|l|}{ Protocol } & $\mathbf{A}$ & $\mathbf{B}$ & C & D & $\mathbf{E}$ & $\mathbf{F}$ \\
\hline \multicolumn{2}{|l|}{ Washing step } & $10 \times$ PBST & $30-35 \times$ PBST & 4-16x Gly-HCl & $10-35 \times$ PBST & $4 \times$ Gly-HCl & $4 \times$ Gly $-\mathrm{HCl}$ \\
\hline \multirow{2}{*}{\multicolumn{2}{|c|}{ Elution step }} & \multirow{2}{*}{$\begin{array}{l}\text { Streptavidin } \\
100 \mu \mathrm{g} / \mathrm{mL} 1 \mathrm{~h}\end{array}$} & \multirow{2}{*}{$\begin{array}{l}8-10 \times \text { Gly-HCl } \\
5 \mathrm{~min}\end{array}$} & \multirow{2}{*}{$\begin{array}{l}\text { Bacterial host } \\
\left(\mathrm{OD}_{600} 0.1\right) 1 \mathrm{~h}\end{array}$} & 4-10× Gly-HCl & \multirow{2}{*}{$\begin{array}{l}\text { Gly- } \mathrm{HCl} \\
\text { Sonication } \\
10 \text { min }\end{array}$} & \multirow{2}{*}{$\begin{array}{l}0.1 \mathrm{mM} \text { biotin } \\
1 \mathrm{~h}\end{array}$} \\
\hline & & & & & $\begin{array}{l}\text { Bacterial host } \\
\left(O_{600} 0.1\right) 1 \mathrm{~h}\end{array}$ & & \\
\hline \multicolumn{2}{|c|}{ No. of selection rounds } & 3 & 3 & 3 & $1-5$ & $1-3$ & 3 \\
\hline \multirow{2}{*}{$\begin{array}{l}\text { Average value } \\
\text { from ELISA } \\
\text { assaya }\end{array}$} & Streptavidin & $0.43 \pm 0.05$ & $0.36 \pm 0.02$ & $0.32 \pm 0.11$ & $0.49 \pm 0.10$ & $0.72 \pm 0.20$ & $1.36 \pm 0.43$ \\
\hline & Blank & $0.26 \pm 0.03$ & $0.16 \pm 0.04$ & $0.16 \pm 0.05$ & $0.29 \pm 0.06$ & $0.11 \pm 0.02$ & $0.21 \pm 0.08$ \\
\hline
\end{tabular}




\section{ArrayStar shines at straightforward microarray
analysis}

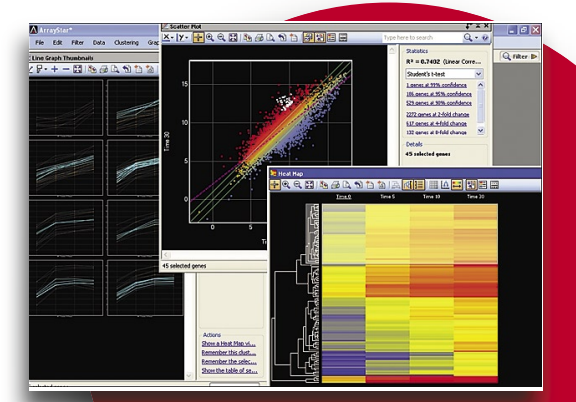

A selection of genes highlighted within simultaneous multiple views of ArrayStar

You benefit from these features and more!

- Compatible with Affymetrix and NimbleGen soffware

- Works seamlessly with .txt files

- Conducts a wide range of analyses and visualizations to evaluate expression patterns

- Clusters images to identify groups of co-regulated genes

- Plots gene expression levels to view relationships with line Graphs and Thumbnails

To receive a FREE fully-functional trial version visit our website or contact us today.

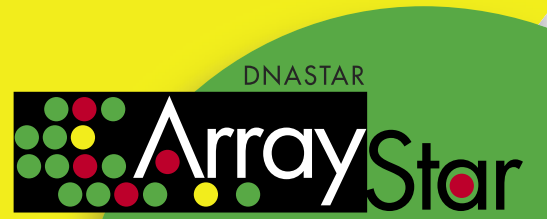

www.dnastar.com

DNASTAR, Inc

Madison, WI, USA

608-258-7420

Toll free: 866-51 1-5090 out. Following 10 -fold washing with $0.2 \%$ PBST, phages were eluted with $100 \mu \mathrm{l}$ of $0.1 \mathrm{mM}$ biotin for $1 \mathrm{~h}$ at $100 \mathrm{rpm}$.

\section{ELISA of Phage Clones}

Microtiter plate wells were coated with $100 \mu \mathrm{l}$ of streptavidin solution $(20 \mu \mathrm{g} / \mathrm{mL})$ in $50 \mathrm{mM} \mathrm{NaHCO}_{3}, \mathrm{pH} 8.5$, overnight and blocked with $200 \mu$ l blocking buffer $(2 \%$ BSA [Sigma-Aldrich] in PBS) for $1 \mathrm{~h}$. A separate set of wells was blocked with blocking buffer without previous streptavidin immobilization as negative controls. One hundred microliters of each selected amplified phage clone were diluted to 200 $\mu \mathrm{l}$ with blocking buffer and transferred to coated wells. Next, the ELISA assay was performed as previously described (9).

\section{DNA Sequencing}

When the ELISA signal of individual clones exceeded the blank by at least fourfold, displayed peptide sequences were determined. Single-stranded DNA from amplified selected phage clones was isolated and sequenced as previously described (9).

\section{Efficacy of Phage Desorption}

Microtiter plates precoated and preblocked with streptavidin were incubated with $4 \times 10^{10} \mathrm{pfu}$ of clone CGTFAHPQC, diluted to $100 \mu \mathrm{l}$ with $0.1 \%$ PBST for $1 \mathrm{~h}$ at room temperature with gentle agitation. Wells were then washed 10 times with $0.2 \%$ PBST and eluted using different elution strategies as described in Protocols A, B, C, E, and F. Wells were washed once again with $0.1 \%$ PBST and incubated with anti-M13 monoclonal antibody. Subsequently, ELISA was performed as previously described (9).

\section{Efficacy of Target Desorption}

Microtiter plates precoated and preblocked with streptavidin were washed 10 times with $0.2 \%$ PBST and eluted using either:

a. neutral-pH (PBS) as control

b. low-pH (0.2 M glycine-HCl buffer, $\mathrm{pH} 2.2)$

c. low-pH (0.2 M glycine-HCl buffer, $\mathrm{pH}$ 2.2) and sonication

Next, wells were incubated with an aliquot of $5 \times 10^{9}$ pfu of clone

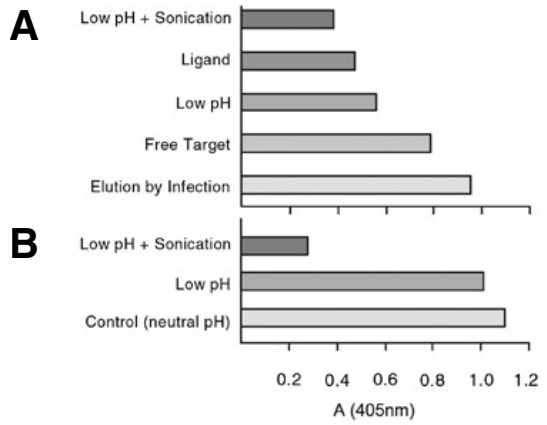

Figure 2. Efficacy of phage and target desorption. (A) The isolated high-affinity phage clone displaying cyclic peptide CGTFAHPQC was incubated over immobilized streptavidin, and the complex was washed with PBST and eluted by different methods. The amount of non-eluted phages was detected by ELISA. Combination of low $\mathrm{pH}$ and sonication removed bound virions most effectively as indicated by lowest absorbance at $405 \mathrm{~nm}$. (B) To investigate target desorption the microtiter plate surface with immobilized streptavidin was exposed to low $\mathrm{pH}$ or a combination of low $\mathrm{pH}$ and sonication. The residual target was detected using the phage clone displaying the peptide CGTFAHPQC and the ELISA assay as above. Intact surface with no elution of bound phages (incubation in neutral PBS buffer) was used for control for both experiments. Combination of low $\mathrm{pH}$ and sonication effectively removed immobilized streptavidin from the microtiter plate.

CGTFAHPQC, diluted to $100 \mu \mathrm{l}$ with $0.1 \%$ PBST, and mixed with $100 \mu \mathrm{l}$ of blocking buffer for $1 \mathrm{~h}$ at room temperature with gentle agitation. Wells were washed three times with $0.1 \%$ PBST and incubated with anti-M13 monoclonal antibody. Subsequently, ELISA was performed as described (9).

\section{Efficacy of Antigen-Antibody Complex Disruption}

Rabbit polyclonal antibody against cathepsin B, purified from antisera (12), was adsorbed to a microtiter plate, to which cathepsin B (67.2, 38.1, and 19.05 $\mathrm{ng} / \mathrm{mL}$ ), produced at Jozef Stefan Institute (13), was added. Cathepsin B was eluted either with:

a. low-pH buffer $(0.2 \mathrm{M}$ glycine-HCl buffer, $\mathrm{pH}$ 2.2)

b. low-pH buffer $(0.2 \mathrm{M}$ glycine-HCl buffer, $\mathrm{pH}$ 2.2) and sonication

c. neutral-pH buffer (PBS) as control

The eluates were saved for analysis. Residual cathepsin B was subsequently detected in a sandwich ELISA assay with secondary sheep anti-cathepsin B antibody (12) conjugated with horseradish 


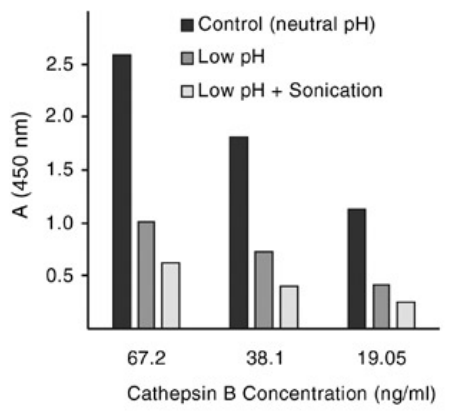

Figure 3. Effect of sonication on a model antigen-antibody complex. Polyclonal antibody against cathepsin B was adsorbed to a microtiter plate to which three different concentrations of cathepsin B were added. The amount of cathepsin $\mathrm{B}$ remaining on the microtiter plate well surface after elution at low $\mathrm{pH}$ or a combination of low $\mathrm{pH}$ and sonication was determined in a sandwich ELISA assay with secondary sheep anti-cathepsin B antibody conjugated with horseradish peroxidase. The most effective elution was achieved by using a combination of low-pH buffer and sonication. Incubation with neutral $\mathrm{pH}$ (PBS) represents the control conditions.

peroxidase. After $2 \mathrm{~h}$ of incubation at $37^{\circ} \mathrm{C}$, peroxidase substrate $3,3^{\prime}, 5,5^{\prime}$-tetramethylbenzidine (TMB; Sigma Chemical Co., St. Louis, MO, USA) in the presence of hydrogen peroxide was added. The amount of degraded substrate, as a measure of bound immuno-complexed cathepsin B, was determined by absorbance at $450 \mathrm{~nm}$.

Eluates from previous experiments were adjusted to neutral $\mathrm{pH}$ (if appropriate) and the proteins allowed to adsorb to a new set of microtiter plate wells. Both cathepsin B and polyclonal antibody against cathepsin B were determined in ultrasound eluates with horseradish peroxidase-conjugated anti-cathepsin $\mathrm{B}$ antibody or anti-rabbit antibody, respectively, using TMB and measuring absorbance at $450 \mathrm{~nm}$.

\section{RESULTS AND DISCUSSION}

We used streptavidin, immobilized by adsorption, as a model target protein for affinity selection of peptides from a lowavidity type 3 phage display library (i.e., a maximum of five copies of peptides are displayed per virion as fusions to coat protein pIII). Biotin was used for competitive elution (Table 1, Protocol F) because of its extremely strong interaction with streptavidin $\left(\mathrm{K}_{\mathrm{d}} \sim 10^{-15} \mathrm{M}\right)$ (14) and because of well-characterized peptide (HPQ) biotin mimotopes $(3,15)$.
In addition, the selection efficiency of a number of well-known nonspecific elution strategies (Table 1, Protocols A-D) was compared with that of biotin elution (Table 1, Protocol F). Individual clones obtained by different elution strategies were tested for their affinity to streptavidin (Figure 1).

Phage-displayed peptide sequences were determined if the ELISA signal of individual clones exceeded the blank by at least fourfold. Such phage clones displayed peptide CGTFAHPQC and were obtained only in selection Protocols $\mathrm{E}$ and $\mathrm{F}$. In all other selection protocols, ELISA signals exceeded the blank by twofold or less, therefore binding was considered to be target-unrelated or not selective. Determination of displayed peptide primary structures of two phage clones with the highest test/blank ratio from Protocol D revealed peptide CQPPGPYSC, which lacks the HPQ mimotope.

The most commonly used low-pH elution is in most cases sufficient for successful selection $(6,16)$. However, in the case of streptavidin, the consensus sequence is not likely to be obtained, as stated by the library manufacturer (http:// www.neb.com/nebecomm/products/ faqproductE8120.asp\#619).

Indeed protocols involving elution with glycine-HCl buffer (Protocols B and D) were incapable of selecting for the HPQ tripeptide motif, even with repetitive elution steps. This could not be attributed to a too stringent washing procedure, since competitive elution with biotin (Protocol F) under similar conditions was effective, nor to unfinished selection, since HPQbearing clones were no longer present in the enriched libraries after the third selection round in Protocols A, B, and C. This suggests that none of the nonspecific elution procedures used so far are able to retrieve strongly bound phages.

Here we report on an improved nonspecific elution method (Table 1, Protocol E), capable of selecting high-affinity clones. The crucial modification is the introduction of ultrasound during elution at low $\mathrm{pH}$. Combined with rigorous washing, this technique achieved efficient selection of a single clone displaying the anticipated HPQ tripeptide in as little as one selection round. If a larger diversity of peptide ligands is desired, the washing stringency should be lowered. Despite rigorous washing and elution conditions, phages have been found to remain sufficiently unimpaired to allow further proliferation.
The efficiencies of all the elution strategies in general use in retrieving a phage clone displaying the cyclic peptide CGTFAHPQC from a streptavidin-coated surface were compared quantitatively (Figure 2A). Ultrasound elution proved the most effective, followed by competitive elution with biotin, low-pH elution, free target, and direct bacterial infection, in that order.

In theory, retrieval of virions can be achieved either by breaking strong ligandtarget interactions or by desorption of the immobilized target. Figure 2B shows that the use of ultrasound during elution in low-pH buffer contributes significantly to target protein desorption, enabling efficient detachment of phages that would otherwise remain bound to the target, despite the lowered $\mathrm{pH}$ (that is, the best binders).

Finally, we confirmed the efficacy and mechanism of ultrasound elution using a model interaction of cathepsin $\mathrm{B}$ and rabbit anti-cathepsin B polyclonal antibody (Figure 3). The antibody was adsorbed to a microtiter plate and cathepsin B was added. The amount of cathepsin $B$ remaining after elution on the microtiter plate well surface was determined using a secondary antibody conjugated to horseradish peroxidase. The ELISA signal intensity showed that the cathepsin $\mathrm{B}$ remaining after sonication at low $\mathrm{pH}$ was, on average, $41 \%$ of that remaining after low-pH elution alone. Moreover, ultrasound elution removed cathepsin $\mathrm{B}$ as well as the adsorbed capturing antibody, since both were detected in the eluate. Thus, sonication clearly aids elution by target desorption, although its possible contribution to direct breaking of ligandtarget interaction cannot be excluded.

In conclusion, all the commonly used elution strategies have failed to elute and select high-affinity HPQ-bearing phage clones bound to streptavidin. The failure was shown to be due to the inability of eluants to break the interaction of highaffinity clones with the target, which is thus likely to be the cause for failed selection with other targets as well. To surmount this, we have introduced a new elution strategy, combining low-pH elution buffer with sonication, which, in addition to loosening the peptide-target interaction, also serves to detach the target molecule from the immobilization surface. This ultrasound-based method enables singlestep selection of a high-affinity peptide from a library of diversity $>10^{9}$, and thus 
